\title{
Linear $q$-Difference Equations
}

\author{
M. H. Abu Risha, M. H. Annaby, M. E. H. Ismail \\ and Z. S. Mansour
}

\begin{abstract}
We prove that a linear $q$-difference equation of order $n$ has a fundamental set of $n$-linearly independent solutions. A $q$-type Wronskian is derived for the $n$ th order case extending the results of Swarttouw-Meijer (1994) in the regular case. Fundamental systems of solutions are constructed for the $n$-th order linear $q$-difference equation with constant coefficients. A basic analog of the method of variation of parameters is established.
\end{abstract}

Keywords. $q$-Difference equations, $q$-Wronskian, $q$-type Liouville's formula

Mathematics Subject Classification (2000). Primary 39A13, secondary 15A03, $34 \mathrm{~A} 30$

\section{Introduction and basic definitions}

In the following, $q$ is a positive number, $0<q<1$, and $I$ is an open interval containing zero. Now we state the basic definitions used in this article, cf. [4, 9]. Then we introduce a brief account about the $q$-calculus established in [3]. Let $n \in \mathbb{N}$. The $q$-shifted factorial $(a ; q)_{n}$ of $a \in \mathbb{C}$ is defined by

$$
(a ; q)_{0}:=1 \quad \text { and, for } n>0, \quad(a ; q)_{n}:=\prod_{k=1}^{n}\left(1-a q^{k-1}\right) .
$$

The multiple $q$-shifted factorial for complex numbers $a_{1}, \ldots, a_{k}$ is defined by

$$
\left(a_{1}, a_{2}, \ldots, a_{k} ; q\right)_{n}:=\prod_{j=1}^{k}\left(a_{j} ; q\right)_{n} .
$$

M. H. Abu Risha, M. H. Annaby, Z. S. Mansour: Department of Mathematics, Cairo University, Faculty of Science, Giza, Egypt;

moemenha@yahoo.com,mhannaby@yahoo.com, zeinabs98@hotmail.com

M. E. H. Ismail: Department of Mathematics, University of Central Florida, Orlando, Florida 33620-5700; ismail@math.ucf.edu 
The $\operatorname{limit}_{n \rightarrow \infty}(a ; q)_{n}$ exists and is denoted by $(a ; q)_{\infty}$. The third type of the $q$-Bessel functions of Jackson of order $\nu$ is defined to be, see [10],

$$
J_{\nu}^{(3)}(x ; q)=x^{\nu} \frac{\left(q^{\nu+1} ; q\right)_{\infty}}{(q ; q)_{\infty}} \sum_{n=0}^{\infty}(-1)^{n} q^{\frac{n(n+1)}{2}} \frac{x^{2 n}}{(q ; q)_{n}\left(q^{\nu+1} ; q\right)_{n}} .
$$

We denote this function by $J_{\nu}(x ; q)$ instead of $J_{\nu}^{(3)}(x ; q)$ for simplicity. In some literature this function is called the Hahn-Exton $q$-Bessel function, see [12, 14]. The functions $\cos _{q} x$ and $\sin _{q} x$ are defined for $x \in \mathbb{C},|x|(1-q)<1$, by

$$
\begin{aligned}
\cos _{q} x & :=\sum_{n=0}^{\infty}(-1)^{n} \frac{(x(1-q))^{2 n}}{(q ; q)_{2 n}} \\
\sin _{q} x & :=\sum_{n=0}^{\infty}(-1)^{n} \frac{(x(1-q))^{2 n+1}}{(q ; q)_{2 n+1}} .
\end{aligned}
$$

The functions $\cos (x ; q)$ and $\sin (x ; q)$ are defined in $\mathbb{C}$ by

$$
\begin{aligned}
\cos (x ; q) & :=\sum_{n=0}^{\infty}(-1)^{n} \frac{q^{n^{2}}(x(1-q))^{2 n}}{(q ; q)_{2 n}} \\
\sin (x ; q) & :=\sum_{n=0}^{\infty}(-1)^{n} \frac{q^{n(n+1)}(x(1-q))^{2 n+1}}{(q ; q)_{2 n+1}}
\end{aligned}
$$

and they are $q$-analogs of the sine and cosine functions, [4, 9]. See also [1, 2], [5]-[7] for a study of the zeros and completeness of $q$-trigonometric and $q$-Bessel systems.

Let $\mu \in \mathbb{R}$ be fixed. A set $A \subseteq \mathbb{R}$ is called a $\mu$-geometric set if for $x \in A$, $\mu x \in A$. Now, we define the $q$-difference operator of Heine. Let $f$ be a function defined on a $q$-geometric set $A \subseteq \mathbb{R}$. The $q$-difference operator is defined by the formula

$$
D_{q} f(x):=\frac{f(x)-f(q x)}{x-q x}, \quad x \in A \backslash\{0\} .
$$

If $0 \in A$, we say that $f$ has $q$-derivative at zero if the limit

$$
\lim _{n \rightarrow \infty} \frac{f\left(x q^{n}\right)-f(0)}{x q^{n}}, \quad x \in A
$$

exists and does not depend on $x$. In this case, we shall denote this limit by $D_{q} f(0)$. In some literature the $q$-derivative at zero is defined to be $f^{\prime}(0)$ if it exists, cf. $[12,14]$, but the above definition is more suitable for our approach. The non-symmetric Leibniz' rule

$$
D_{q}(f g)(x)=g(x) D_{q} f(x)+f(q x) D_{q} g(x)
$$


holds. Relation (1.1) can be symmetrized using the relation $f(q x)=f(x)-x(1-$ q) $D_{q} f(x)$, giving the additional term $-x(1-q) D_{q} f(x) D_{q} g(x)$. The $q$-integration of F. H. Jackson [11] is defined for a function $f$ defined on a $q$-geometric set $A$ to be

$$
\int_{a}^{b} f(t) d_{q} t=\int_{0}^{b} f(t) d_{q} t-\int_{0}^{a} f(t) d_{q} t, \quad a, b \in A,
$$

where

$$
\int_{0}^{x} f(t) d_{q} t=\sum_{n=0}^{\infty} x q^{n}(1-q) f\left(x q^{n}\right), \quad x \in A,
$$

provided that the series converge.

Theorem 1.1 ([3]). The q-integral (1.2) exists only if $\lim _{k \rightarrow \infty} x q^{k} f\left(x q^{k}\right)=0$.

Consider the non-homogeneous $q$-difference equation of order $n$

$$
a_{0}(x) D_{q}^{n} y(x)+a_{1}(x) D_{q}^{n-1} y(x)+\cdots+a_{n}(x) y(x)=b(x), \quad x \in I,
$$

for which $a_{i}, 0 \leqslant i \leqslant n$, and $b$ are continuous at zero functions defined on $I$ and $a_{0}(x) \neq 0$ for all $x \in I$. Equation (1.3) together with the initial conditions

$$
D_{q}^{i-1} y(0)=b_{i}, \quad b_{i} \in \mathbb{C}, i=1, \ldots, n,
$$

form a $q$-type Cauchy problem. By a solution of problem (1.3)-(1.4), we mean a continuous at zero function which satisfies (1.3) subject to the initial conditions (1.4). According to [3], there exists a unique solution of (1.3)-(1.4) in a subinterval $J$ of $I, J=[-h, h], h>0$. In the next section, we shall study the $n$-th order homogeneous linear equation

$$
a_{0}(x) D_{q}^{n} y(x)+a_{1}(x) D_{q}^{n-1} y(x)+\cdots+a_{n}(x) y(x)=0, \quad x \in I .
$$

A fundamental set of solutions for (1.5) when the coefficients are constants is derived in $\S 2$. In $\S 3$, a $q$-type Wronskian for the solutions of (1.5) is introduced and it is proved that it satisfies a first order $q$-difference equation and its solution is given. This extends the results of Swarttouw-Meijer [15] in the regular case. As applications, a formula for a solution of (1.3) in terms of a fundamental set of solutions of (1.5) will be given in $\S 4$ by using a $q$-analog of the method of variation of parameters.

\section{Linear homogeneous $q$-difference equations}

Let $M$ denote the set of solutions of (1.5) valid in a subset $J \subseteq I$ which contains zero. Then it is easy to see that $M$ is a linear space over $\mathbb{C}$. Also from the existence and uniqueness of the solutions, cf. [3], if $\phi \in M$ and $D_{q}^{i} \phi(0)=0$, $0 \leqslant i \leqslant n-1$, then $\phi(x) \equiv 0$ on $J$. Moreover, cf. [3], $\left\{D_{q}^{i} \phi\right\}_{i=0}^{n-1}, 0 \leqslant i \leqslant n-1$, are continuous at zero for any $\phi \in M$. A set of $n$ solutions of (1.5) is said to 
be a fundamental set (f.s.) for (1.5) valid in $J$ or a f.s. of $M$ if it is linearly independent in $J$. Moreover, as in differential equations, if $b_{i j}, 1 \leq i, j \leq n$, are numbers, and, for each $j, \phi_{j}$ is the unique solution of (1.5) which satisfies the initial conditions

$$
D_{q}^{i-1} \phi_{j}(0)=b_{i j}, \quad 1 \leq i \leq n,
$$

then $\left\{\phi_{j}\right\}_{j=1}^{n}$ is a f.s. of $(1.5)$ if and only if $\operatorname{det}\left(b_{i j}\right) \neq 0$. Hence $M$ is a linear space of dimension $n$. In the following we are concerned with constructing a f.s. for (1.5) when it has constant coefficients, $a_{r}, 0 \leqslant r \leqslant n$. Set $L:=$ $a_{0} D_{q}^{n}+a_{1} D_{q}^{n-1}+\cdots+a_{n}$. Then, (1.5) can be written as

$$
L y(x)=a_{0} D_{q}^{n} y(x)+a_{1} D_{q}^{n-1} y(x)+\cdots+a_{n} y(x)=0 .
$$

The characteristic polynomial $P(\lambda)$ of $(2.1)$ is defined by

$$
P(\lambda)=a_{0} \lambda^{n}+a_{1} \lambda^{n-1}+\cdots+a_{n}, \quad \lambda \in \mathbb{C} .
$$

Let $\lambda_{i}, 1 \leqslant i \leqslant k$, denote the distinct roots of $P(\lambda)$ and $m_{i}$ denotes the multiplicity of $\lambda_{i}$, so that $\sum_{i=1}^{k} m_{i}=n$. Corresponding to each $\lambda_{i}$ we define an $m_{i}$-dimensional subspace $M_{i}$ by

$$
M_{i}=\left\{v \in M:\left(D_{q}-\lambda_{i}\right)^{m_{i}} v=0\right\} .
$$

The construction of a f.s. of (2.1) depends on the fact that, cf. [13],

$$
M=M_{1} \oplus \cdots \oplus M_{k} .
$$

Lemma 2.1. Let $(X, \mathbb{K})$ be a vector space, and let $T$ be a linear operator on $X$. For any $\lambda \in \mathbb{K}$, if there exist $y_{0}, y_{1}, \ldots, y_{m-1}$ in $X$ such that

$$
\begin{array}{ll}
T y_{0}=\lambda y_{0}, & y_{0} \neq 0 \\
T y_{i}=\lambda y_{i}+y_{i-1}, & 1 \leqslant i \leqslant m-1,
\end{array}
$$

then $y_{1}, \ldots, y_{m-1}$ are linearly independent.

Proof. By induction on $i, 0 \leqslant i \leqslant m-1$.

Lemma 2.2. If $\lambda_{i} \neq 0$, then the initial value problem

$$
\begin{aligned}
D_{q} \phi_{0, i} & =\lambda_{i} \phi_{0, i}, & \phi_{0, i}(0) & =1, r \\
D_{q} \phi_{r, i} & =\lambda_{i} \phi_{r, i}+\phi_{r-1, i}, & \phi_{r, i}(0) & =0, \quad r=1, \ldots, m_{i}-1,
\end{aligned}
$$

has the solution

$$
\phi_{r, i}(x)= \begin{cases}e_{q}\left(\lambda_{i} x\right):=\sum_{k=0}^{\infty} \frac{\left(\lambda_{i} x(1-q)\right)^{k}}{(q ; q)_{k}}, & r=0 \\ \frac{1}{\lambda_{i}^{r}} \sum_{k=r}^{\infty} \frac{k(k-1) \cdots(k-r+1)}{r !} \frac{\left(\lambda_{i} x(1-q)\right)^{k}}{(q ; q)_{k}}, & r=1,2, \ldots, m_{i}-1,\end{cases}
$$

which is valid for $|x|<\frac{1}{\lambda_{i}(1-q)}$. If $\lambda_{i}=0$, then

$$
\phi_{r, i}(x)=\frac{x^{r}(1-q)^{r}}{(q ; q)_{r}}, \quad r=0,1, \ldots, m_{i}-1 .
$$


Proof. The proof follows by direct computations.

One can see that $\left(D_{q}-\lambda_{i}\right)^{m_{i}} \phi_{r, i}=0, r=0,1, \ldots, m_{i}-1$. Thus, $\phi_{r, i} \in M_{i}$, for $r=0,1, \ldots, m_{i}-1$. Therefore, these functions form a basis for $M_{i}$ since they are linearly independent by Lemma 2.1. This fact and (2.2) above imply the following theorem.

Theorem 2.3. The set $\left\{\phi_{i, r}\right\}_{r=0}^{m_{i}-1}$ of (2.3) when $\lambda_{i} \neq 0$ or of (2.4) when $\lambda_{i}=0$ is a linearly independent set of solutions of (2.1). Moreover, $\bigcup_{i=1}^{k}\left\{\phi_{i, r}\right\}_{r=0}^{m_{i}-1}$ is a fundamental set of solutions of (2.1).

Example 2.4. The $q$-difference equation

$$
D_{q}^{3} y(x)-4 D_{q}^{2} y(x)+5 D_{q} y(x)-2 y(x)=0,
$$

has the functions $e_{q}(2 x), e_{q}(x)$ and $\sum_{k=1}^{\infty} k \frac{(x(1-q))^{k}}{(q ; q)_{k}}$ as a f.s..

\section{A q-type Wronskian}

This section contains a $q$-analog of the Wronskian of linear differential equations, we prove that the $q$-analog satisfies a first order $q$-difference equation and we derive its solution. We also derive a $q$-type Liouville's formula for the $q$-Wronskian.

Definition 3.1. Let $y_{i}, 1 \leq i \leq n$, be functions defined on a $q$-geometric set $A$. The $q$-Wronskian of the functions $y_{i}$ which will be denoted by $W_{q}\left(y_{1}, \ldots, y_{n}\right)(x)$ is defined to be

$$
W_{q}\left(y_{1}, \ldots, y_{n}\right)(x):=\left|\begin{array}{ccc}
y_{1}(x) & \cdots & y_{n}(x) \\
D_{q} y_{1}(x) & \cdots & D_{q} y_{n}(x) \\
\vdots & \ddots & \vdots \\
D_{q}^{n-1} y_{1}(x) & \cdots & D_{q}^{n-1} y_{n}(x)
\end{array}\right|
$$

provided that the derivatives exist in $I$. For convenience we write $W_{q}(x)$ instead of $W_{q}\left(y_{1}, \ldots, y_{n}\right)(x)$.

Lemma 3.2. Let $y_{1}, \ldots, y_{n}$ be functions defined on a q-geometric set $A$. Then for any $x \in A, x \neq 0$,

$D_{q} W_{q}\left(y_{1}, y_{2}, \ldots, y_{n}\right)(x)=\left|\begin{array}{cccc}y_{1}(q x) & y_{2}(q x) & \cdots & y_{n}(q x) \\ \left(D_{q} y_{1}\right)(q x) & \left(D_{q} y_{2}\right)(q x) & \cdots & \left(D_{q} y_{n}\right)(q x) \\ \vdots & \vdots & \ddots & \vdots \\ \left(D_{q}^{n-2} y_{1}\right)(q x) & \left(D_{q}^{n-2} y_{2}\right)(q x) & \cdots & \left(D_{q}^{n-2} y_{n}\right)(q x) \\ D_{q}^{n} y_{1}(x) & D_{q}^{n} y_{2}(x) & \cdots & D_{q}^{n} y_{n}(x)\end{array}\right|$ 
Proof. We prove the lemma by induction on $n$. The lemma is trivial when $n=1$. Assume that (3.1) holds at $k \in \mathbb{N}, k \geqslant 1$, then expanding $W_{q}\left(y_{1}, y_{2}, \ldots, y_{k+1}\right)$ in terms of the first row we obtain

$$
W_{q}\left(y_{1}, y_{2}, \ldots, y_{k+1}\right)(x)=\sum_{j=1}^{k+1}(-1)^{j+1} y_{j}(x) W_{q}^{(j)}(x)
$$

where

$$
W_{q}^{(j)}:= \begin{cases}W_{q}\left(D_{q} y_{2}, \ldots, D_{q} y_{k+1}\right), & j=1 \\ W_{q}\left(D_{q} y_{1}, \ldots, D_{q} y_{j-1}, D_{q} y_{j+1}, \ldots, D_{q} y_{k+1}\right), & 1 \leqslant j \leqslant k+1 \\ W_{q}\left(D_{q} y_{1}, \ldots, D_{q} y_{k}\right), & j=k+1 .\end{cases}
$$

Consequently, from (1.1),

$$
\begin{aligned}
D_{q} W_{q} & \left(y_{1}, y_{2}, \ldots, y_{k+1}\right)(x) \\
& =\sum_{j=1}^{k+1}(-1)^{j+1} D_{q} y_{j}(x) W_{q}^{(j)}(x)+\sum_{j=1}^{k+1}(-1)^{j+1} y_{j}(q x) D_{q} W_{q}^{(j)}(x) .
\end{aligned}
$$

Now

$$
\sum_{j=1}^{k+1}(-1)^{j+1} D_{q} y_{j}(x) W_{q}^{(j)}(x)=\left|\begin{array}{ccc}
D_{q} y_{1}(x) & \cdots & D_{q} y_{k+1}(x) \\
D_{q} y_{1}(x) & \cdots & D_{q} y_{k+1}(x) \\
D_{q}^{2} y_{1}(x) & \cdots & D_{q}^{2} y_{k+1}(x) \\
\vdots & \ddots & \vdots \\
D_{q}^{k-1} y_{1}(x) & \cdots & D_{q}^{k-1} y_{k+1}(x) \\
D_{q}^{k} y_{1}(x) & \cdots & D_{q}^{k} y_{k+1}(x)
\end{array}\right|=0
$$

and from the induction hypothesis,

$$
\begin{aligned}
& \sum_{j=1}^{k+1}(-1)^{j+1} y_{j}(q x) D_{q} W_{q}^{(j)}(x)=\sum_{j=1}^{k+1}(-1)^{j+1} y_{j}(q x) \times \\
& \left|\begin{array}{cccccc}
\left(D_{q} y_{1}\right)(q x) & \cdots & \left(D_{q} y_{j-1}\right)(q x) & \left(D_{q} y_{j+1}\right)(q x) & \cdots & \left(D_{q} y_{k+1}\right)(q x) \\
\left(D_{q}^{2} y_{1}\right)(q x) & \cdots & \left(D_{q}^{2} y_{j-1}\right)(q x) & \left(D_{q}^{2} y_{j+1}\right)(q x) & \cdots & \left(D_{q}^{2} y_{k+1}\right)(q x) \\
\vdots & \ddots & \vdots & \vdots & \ddots & \vdots \\
\left(D_{q}^{k-1} y_{1}\right)(q x) & \cdots & \left(D_{q}^{k-1} y_{j-1}\right)(q x) & D_{q}^{k-1} y_{j+1}(q x) & \cdots & D_{q}^{k-1} y_{k+1}(x)(q x) \\
D_{q}^{k+1} y_{1}(x) & \cdots & D_{q}^{k+1} y_{j-1}(x) & D_{q}^{k+1} y_{j+1}(x) & \cdots & D_{q}^{k+1} y_{k+1}(x)
\end{array}\right|,
\end{aligned}
$$

where when $i=1$ the determinant of (3.2) starts with $D_{q} y_{2}(q x)$ and when $j=k+1$, the determinant ends with $D_{q}^{k+1} y_{k}(x)$. Thus

$$
\sum_{j=1}^{k+1}(-1)^{j+1} y_{j}(q x) D_{q} W_{q}^{(j)}(x)=\left|\begin{array}{ccc}
y_{1}(q x) & \cdots & y_{k+1}(q x) \\
\left(D_{q} y_{1}\right)(q x) & \cdots & \left(D_{q} y_{k+1}\right)(q x) \\
\left(D_{q}^{2} y_{1}\right)(q x) & \cdots & \left(D_{q}^{2} y_{k+1}\right)(q x) \\
\vdots & \ddots & \vdots \\
\left(D_{q}^{k-1} y_{1}\right)(q x) & \cdots & \left(D_{q}^{k-1} y_{k+1}\right)(q x) \\
D_{q}^{k+1} y_{1}(x) & \cdots & D_{q}^{k+1} y_{k+1}(x)
\end{array}\right|
$$


proving (3.1) for $n=k+1$ and hence all $k \in \mathbb{N}$.

Theorem 3.3. If $y_{1}, y_{2}, \ldots, y_{n}$ are solutions of (1.5) in $J \subseteq I$, then their $q$-Wronskian satisfies the first order q-difference equation

$$
\begin{aligned}
D_{q} W_{q}(x) & =-R(x) W_{q}(x), \quad x \in J \backslash\{0\} \\
R(x) & =\sum_{k=0}^{n-1}(x-q x)^{k} \frac{a_{k+1}(x)}{a_{0}(x)} .
\end{aligned}
$$

Proof. From the definition of the operator $D_{q}$, we have

$$
\left(D_{q}^{m} y\right)(q x)=D_{q}^{m} y(x)-x(1-q) D_{q}^{m+1} y(x), \quad m \in \mathbb{N} .
$$

Substituting in (3.1) yields

$$
\begin{aligned}
& D_{q} W_{q}\left(y_{1}, \ldots, y_{n}\right)(x) \\
& =\left|\begin{array}{ccc}
y_{1}(x)-x(1-q) D_{q} y_{1}(x) & \cdots & y_{n}(x)-x(1-q) D_{q} y_{n}(x) \\
D_{q} y_{1}(x)-x(1-q) D_{q}^{2} y_{1}(x) & \cdots & D_{q} y_{n}(x)-x(1-q) D_{q}^{2} y_{n}(x) \\
\vdots & \ddots & \vdots \\
D_{q}^{n-2} y_{1}(x)-x(1-q) D_{q}^{n-1} y_{1}(x) & \cdots & D_{q}^{n-2} y_{n}(x)-x(1-q) D_{q}^{n-1} y_{n}(x) \\
D_{q}^{n} y_{1}(x) & \cdots & D_{q}^{n} y_{n}(x)
\end{array}\right| .
\end{aligned}
$$

We shall prove by induction on $n$ that

$$
\begin{aligned}
& D_{q} W_{q}\left(y_{1}, \ldots, y_{n}\right)(x) \\
& =\sum_{k=1}^{n}(-1)^{k-1}(x-q x)^{k-1}\left|\begin{array}{ccc}
y_{1}(x) & \cdots & y_{n}(x) \\
D_{q} y_{1}(x) & \cdots & D_{q} y_{n}(x) \\
\vdots & \ddots & \vdots \\
D_{q}^{n-k-1} y_{1}(x) & \cdots & D_{q}^{n-k-1} y_{n}(x) \\
D_{q}^{n-k+1} y_{1}(x) & \cdots & D_{q}^{n-k+1} y_{n}(x) \\
\vdots & \ddots & \vdots \\
D_{q}^{n} y_{1}(x) & \cdots & D_{q}^{n} y_{n}(x)
\end{array}\right| .
\end{aligned}
$$

If (3.4) holds at $n=m$, then

$$
D_{q} W_{q}\left(y_{1}, y_{2}, \ldots, y_{m+1}\right)(x)=\sum_{j=1}^{m+1}(-1)^{j+1}\left(y_{j}(x)-x(1-q) D_{q} y_{j}(x)\right) A_{1 j},
$$

where

$$
A_{1 j}=D_{q} W_{q}\left(D_{q} y_{1}, \ldots, D_{q} y_{j-1}, D_{q} y_{j+1}, \ldots, D_{q} y_{m+1}\right), \quad j=1,2, \ldots, m .
$$


Hence from the previous hypothesis we obtain

$$
\begin{aligned}
& D_{q} W_{q}\left(y_{1}, y_{2}, \ldots, y_{m+1}\right)(x) \\
& =\sum_{j=1}^{m+1}(-1)^{j+1}\left(y_{j}(x)-x(1-q) D_{q} y_{j}(x)\right) \sum_{k=1}^{m}(-1)^{k-1}(x(1-q))^{k-1} B_{j k} \\
& =\sum_{k=1}^{m}(-1)^{k-1}(x(1-q))^{k-1} \sum_{j=1}^{m+1}(-1)^{j+1} y_{j}(x) B_{j k} \\
& \quad+\sum_{k=1}^{m}(-1)^{k}(x(1-q))^{k} \sum_{j=1}^{m+1}(-1)^{j+1} D_{q} y_{j}(x) B_{j k},
\end{aligned}
$$

with

$$
B_{j k}:=\left|\begin{array}{cccccc}
D_{q} y_{1}(x) & \cdots & D_{q} y_{j-1}(x) & D_{q} y_{j}(x) & \cdots & D_{q} y_{m+1}(x) \\
D_{q}^{2} y_{1}(x) & \cdots & D_{q}^{2} y_{j-1}(x)(x) & D_{q}^{2} y_{j}(x) & \cdots & D_{q}^{2} y_{m+1}(x) \\
\vdots & \ddots & \vdots & \vdots & \ddots & \vdots \\
D_{q}^{m-k} y_{1}(x) & \cdots & D_{q}^{m-k} y_{j-1}(x) & D_{q}^{m-k} y_{j}(x) & \cdots & D_{q}^{m-k} y_{m+1}(x) \\
D_{q}^{m-k+2} y_{1}(x) & \cdots & D_{q}^{m-k+2} y_{j-1}(x) & D_{q}^{m+k+1} y_{j+1}(x) & \cdots & D_{q}^{m+k+1} y_{m+1}(x) \\
\vdots & \ddots & \vdots & \vdots & \ddots & \vdots \\
D_{q}^{m+1} y_{1}(x) & \cdots & D_{q}^{m+1} y_{j-1}(x) & D_{q}^{m+1} y_{j+1}(x) & \cdots & D_{q}^{m+1} y_{m+1}(x)
\end{array}\right|,
$$

$k=1,2, \ldots, m$, where when $j=1$ the determinant $B_{1 k}$ start with $D_{q} y_{2}(x)$ and when $j=m+1$, the determinant $B_{(m+1) k}$ ends with $D_{q}^{m+1} y_{m}(x)$. From the properties of the determinants we conclude that

$$
\sum_{j=1}^{m+1}(-1)^{j+1} y_{j}(x) B_{j k}=\left|\begin{array}{ccc}
y_{1}(x) & \cdots & y_{m+1}(x) \\
D_{q} y_{1}(x) & \cdots & D_{q} y_{m+1}(x) \\
\vdots & \ddots & \vdots \\
D_{q}^{m-k} y_{1}(x) & \cdots & D_{q}^{m-k} y_{m+1}(x) \\
D_{q}^{m-k+2} y_{1}(x) & \cdots & D_{q}^{m-k+2} y_{m+1}(x) \\
\vdots & \ddots & \vdots \\
D_{q}^{m+1} y_{1}(x) & \cdots & D_{q}^{m+1} y_{m+1}(x)
\end{array}\right|
$$

$$
\sum_{j=1}^{m+1}(-1)^{j+1} D_{q} y_{j}(x) B_{j k}=0, \quad \text { for } k=1,2, \ldots, m-1
$$

and

$$
\sum_{j=1}^{m+1}(-1)^{j+1} D_{q} y_{j}(x) B_{j m}=\left|\begin{array}{ccc}
D_{q} y_{1}(x) & \cdots & D_{q} y_{m+1}(x) \\
D_{q}^{2} y_{1}(x) & \cdots & D_{q}^{2} y_{m+1}(x) \\
\vdots & \ddots & \vdots \\
D_{q}^{m+1} y_{1}(x) & \cdots & D_{q}^{m+1} y_{m+1}(x)
\end{array}\right|
$$


Combining equations (3.5)-(3.7) with (3), we obtain (3.4) when $n=m+1$. One can easily see that (3.4) holds at $n=1$. Consequently it holds for all $n \in \mathbb{N}$. From (1.5), we have

$$
D_{q}^{n} y_{j}(x)=-\sum_{i=1}^{i=n} \frac{a_{i}(x)}{a_{0}(x)} D_{q}^{n-i} y_{j}(x), \quad j=1,2, \ldots, n .
$$

Then (3.4) is nothing but

$$
D_{q} W_{q}(x)=-\left[\sum_{k=0}^{k=n-1}(x-q x)^{k} \frac{a_{k+1}(x)}{a_{0}(x)}\right] W_{q}(x)=-R(x) W_{q}(x) .
$$

This completes the proof of the theorem.

The next theorem gives a $q$-type Liouville's formula for the $q$-Wronskian.

Theorem 3.4. Let $x(1-q) R(x) \neq-1$ for all $x \in J$. Then the $q$-Wronskian of any set of solutions $\left\{\phi_{i}\right\}_{i=1}^{n}$ of equation (1.5) is given by

$$
\begin{aligned}
W_{q}(x) & =W_{q}\left(\phi_{1}, \ldots, \phi_{n}\right)(x) \\
& =\frac{1}{\prod_{k=0}^{\infty}\left(1+x(1-q) q^{k} R\left(x q^{k}\right)\right)} W_{q}(0), \quad x \in J
\end{aligned}
$$

Proof. Equation (3.3) is

$$
\frac{W_{q}(x)-W_{q}(q x)}{x-q x}=-R(x) W_{q}(x), x \neq 0,
$$

i.e., $W_{q}(x)-W_{q}(q x)=-x(1-q) R(x) W_{q}(x)$. Hence, under the assumption $1+x(1-q) R(x) \neq 0$, we obtain $W_{q}(x)=\frac{W_{q}(q x)}{1+x(1-q) R(x)}$. Therefore,

$$
W_{q}(x)=\frac{W_{q}\left(x q^{m+1}\right)}{\prod_{k=0}^{m}\left(1+x(1-q) q^{k} R\left(x q^{k}\right)\right)}, \quad \text { for all } m \in \mathbb{N} \text { and } x \in I \text {. }
$$

Since all functions $\frac{a_{j}}{a_{0}}$ are continuous at zero, then $\sum_{k=0}^{\infty} q^{k}\left|R\left(x q^{k}\right)\right|$ is convergent. Consequently, $\prod_{k=0}^{\infty}\left(1+x(1-q) q^{k} R\left(x q^{k}\right)\right)$ converges for every $x \in I$. Thus, using the continuity of $W_{q}(x)$ at zero, (3.8) follows.

Corollary 3.5. Let $\left\{\phi_{i}\right\}_{i=1}^{n}$ be a set of solutions of (1.5) in some subinterval $J$ of $I$ which contains zero. Then $W_{q}(x)$ is either never zero or identically zero in I. The first case occurs when $\left\{\phi_{i}\right\}_{i=1}^{n}$ is a fundamental set of (1.5) and the second when it is not. 
Proof. A set of solutions $\left\{\phi_{i}\right\}_{i=1}^{n}$ forms a f.s. of (1.5) if and only if

$$
W_{q}(0)=\operatorname{det}\left(D_{q}^{i-1} \phi_{j}(0)\right)_{i, j=1}^{n} \neq 0
$$

cf. [3]. This proves the corollary since from Theorem $3.4, W_{q}(x) \neq 0$ for all $x \in J$ if and only if $W_{q}(0) \neq 0$.

Example 3.6. In this example we calculate the $q$-Wronskian of

$$
\frac{-1}{q} D_{q^{-1}} D_{q} y(x)+y(x)=0, \quad x \in \mathbb{R} .
$$

The solutions of (3.9) subject to the initial conditions

$$
y(0)=0, D_{q} y(0)=1 \quad \text { and } \quad y(0)=1, D_{q} y(0)=0,
$$

are $\sin (x ; q), \cos (x ; q), x \in \mathbb{R}$, respectively. Since (3.9) can be written as

$$
D_{q}^{2} y(x)+q x(1-q) D_{q} y(x)-q y(x)=0 .
$$

Then $a_{0}(x) \equiv 1, a_{1}(x)=q x(1-q)$ and $a_{2}(x)=-q$. Thus $R(x) \equiv 0$ on $\mathbb{R}$ and $W_{q}(x) \equiv W_{q}(0)$. But

$$
\begin{aligned}
W_{q}(0) & =W_{q}(\cos (\cdot ; q), \sin (\cdot ; q))(0) \\
& =\left.(\cos (x ; q) \cos (\sqrt{q} x ; q)+\sqrt{q} \sin (x ; q) \sin (\sqrt{q} x ; q))\right|_{x=0} \\
& =1
\end{aligned}
$$

Then, $W_{q}(x) \equiv 1$ for all $x \in \mathbb{R}$.

Example 3.7. We calculate the $q$-Wronskian of the solutions of the $q$-difference equations

$$
-D_{q}^{2} y(x)+y(x)=0, \quad x \in \mathbb{R} .
$$

The functions $\sin _{q} x, \cos _{q} x,|x|(1-q)<1$, are solutions of (3.10) subject to the initial conditions

$$
y(0)=0, D_{q} y(0)=1 \quad \text { and } \quad y(0)=1, D_{q} y(0)=0,
$$

respectively. Here $R(x)=x(1-q)$. So, $x(1-q) R(x) \neq-1$ for all $x$ in $\mathbb{R}$. Hence,

$$
W_{q}(x)=\frac{W_{q}(0)}{\prod_{n=0}^{\infty}\left(1+q^{2 n}\{x(1-q)\}^{2}\right)}, \quad|x|(1-q)<1 .
$$

But

$$
W_{q}(0)=W_{q}\left(\cos _{q}, \sin _{q}\right)(0)=\left.\left(\cos _{q}^{2} x+\sin _{q}^{2} x\right)\right|_{x=0}=1 .
$$

Therefore, $W_{q}(x) \equiv\left(\prod_{n=0}^{\infty}\left(1+q^{2 n}\{x(1-q)\}^{2}\right)\right)^{-1},|x|(1-q)<1$. 
Remarks. 1. Theorem 3.3 might be satisfied for less restrictive conditions. But a general treatment needs a separate consideration. The $q$-Wronskian of (1.5) satisfies the first order $q$-difference equation (3.3) whatever the conditions which the functions $a_{j}, 0 \leq j \leq n$ satisfy. But, in this case, the $q$-Wronskian can not be determined by using Theorem 3.4. An example of this case is the second order $q$-difference equation

$$
\begin{aligned}
& q x^{2}(1-q)^{2} D_{q}^{2} y(x)+x(1-q)^{2} D_{q} y(x) \\
& +\left(x^{2} q^{2-\nu}+\left(1-q^{\nu}\right)\left(1-q^{-\nu}\right)\right) y(x q)=0
\end{aligned}
$$

where $\nu>-1$, which has a f.s. $\left\{J_{\nu}\left(x ; q^{2}\right), J_{-\nu}\left(x q^{-\nu} ; q^{2}\right)\right\}$ and it has been treated by R. F. Swarttouw and H. G. Meijer [15]. This class of problems may be considered as singular $q$-difference equations, while we are dealing with regular equations.

2. It is worthy to mention here that if equation (1.5) has the form

$$
a_{0}(x) D_{q}^{n} y(x)+a_{1}(x)\left(D_{q}^{n-1}\right) y(q x)+\cdots+a_{n}(x) y(q x)=0, \quad x \in I,
$$

then substituting with $D_{q}^{n} y(x)=-\sum_{j=1}^{n} \frac{a_{j}(x)}{a_{0}(x)}\left(D_{q}^{n-j} y\right)(q x)$ in (3.1) above we could derive a theory similar to that of the present section. In this case the associated $q$-Wronskian of solutions $z_{1}, \ldots, z_{n}$ of (3.11) will satisfy the simplified first order $q$-difference equation

$$
D_{q} W_{q}(x)=-\frac{a_{1}(x)}{a_{0}(x)} W_{q}(q x) .
$$

Consequently

$$
W_{q}(x)=\prod_{k=0}^{\infty}\left(1-x q^{k}(1-q) \frac{a_{1}\left(x q^{k}\right)}{a_{0}\left(x q^{k}\right)}\right) W_{q}(0), \quad x \in J \backslash\{0\} .
$$

Similar to differential equations if $a_{1} \equiv 0$, then $W_{q}(x)$ is identically a constant. It should be noted that problems involving equation of the form (3.11) plays an important role in defining self adjoint eigenvalue problems, see e.g. [5, 8].

\section{Applications}

The theory introduced in the previous two sections can be used to obtain a general formula for the solutions of the inhomogeneous equation (1.3). Obviously, if $\psi_{1}$ and $\psi_{2}$ are two solutions of (1.3), then $\psi_{1}-\psi_{2}$ is a solution of the corresponding homogeneous equation (1.5). Thus if $\psi$ is a solution of (1.3) and $\left\{\phi_{i}\right\}_{i=1}^{n}$ is a f.s. for (1.5), then there are unique constants $\left\{c_{i}\right\}_{i=1}^{n}$ such that

$$
\psi=c_{1} \phi_{1}+\cdots+c_{n} \phi_{n}+\psi_{0}
$$


where $\psi_{0}$ is a particular solution of (1.3). Now, we introduce a $q$-analog of the method of variation of parameters to find a particular solution $\psi_{0}$ of (1.3). Here also the functions $a_{r}$ and $b$ are continuous at zero functions defined on $I$ such that $a_{0}(x) \neq 0$ for all $x \in I$.

Theorem 4.1. Let $\left\{\phi_{i}\right\}_{i=1}^{n}$ be a fundamental set of (1.5) in J. Then, any solution $\psi$ of (1.3) is given by

$$
\psi(x)=\sum_{i=1}^{n}\left(c_{i}+\int_{0}^{x} \frac{W_{q, i}\left(\phi_{1}, \ldots, \phi_{n}\right)(q t)}{W_{q}\left(\phi_{1}, \ldots, \phi_{n}\right)(q t)} \cdot \frac{b(t)}{a_{0}(t)} d_{q} t\right) \phi_{i}(x),
$$

where the $c_{i}$ 's are constants and $W_{q, r}\left(\phi_{1}, \ldots, \phi_{n}\right)(x)$ is the determinant obtained from $W_{q}\left(\phi_{1}, \ldots, \phi_{n}\right)(x)$ by replacing the $r$-th column by $(0, \ldots, 0,1)$.

Proof. Let $\psi$ be a solution of (1.3). If $\psi_{0}$ is a particular solution of (1.3), then for some constants $c_{1}, c_{2}, \ldots, c_{n}$,

$$
\psi=\psi_{0}+c_{1} \phi_{1}+\cdots+c_{n} \phi_{n}
$$

where $c_{1}, \ldots, c_{n}$ are constants. Assume that $\psi_{0}$ has the form

$$
\psi_{0}(x)=u_{1}(x) \phi_{1}(x)+\cdots+u_{n}(x) \phi_{n}(x),
$$

where $u_{1}, \ldots, u_{n}$ are functions satisfying the system

$$
\begin{aligned}
D_{q} u_{1}(x) \phi_{1}(q x)+\cdots+D_{q} u_{n}(x) \phi_{n}(q x) & =0 \\
D_{q} u_{1}(x) D_{q, q x} \phi_{1}(q x)+\cdots+D_{q} u_{n}(x) D_{q, q x} \phi_{n}(q x) & =0 \\
\vdots & \vdots \\
D_{q} u_{1}(x) D_{q, q x}^{n-2} \phi_{1}(q x)+\cdots+D_{q} u_{n}(x) D_{q, q x}^{n-2} \phi_{n}(q x) & =0 \\
D_{q} u_{1}(x) D_{q, q x}^{n-1} \phi_{1}(q x)+\cdots+D_{q} u_{n}(x) D_{q, q x}^{n-1} \phi_{n}(q x) & =\frac{b(x)}{a_{0}(x)} .
\end{aligned}
$$

System (4.2) is an inhomogeneous linear system of equations in the $n$ unknowns $\left\{D_{q} u_{i}\right\}_{i=1}^{n}$. The determinant of the coefficients is $W_{q}\left(\phi_{1}, \ldots, \phi_{n}\right)(q x) \neq 0$ since $\phi_{r}, 1 \leqslant r \leqslant n$, is a f.s. for (1.5). Hence (4.2) can be solved for the $D_{q} u_{r}$ and

$$
D_{q} u_{r}(x)=\frac{W_{q, r}\left(\phi_{1}, \ldots, \phi_{n}\right)(q x)}{W_{q}\left(\phi_{1}, \ldots, \phi_{n}\right)(q x)} \cdot \frac{b(x)}{a_{0}(x)}, \quad r=1, \ldots, n .
$$

Since $\frac{b}{a_{0}}$ is continuous at zero, then from Theorem $1.1 D_{q} u_{r}$ is $q$-integrable on $[0, x]$, for all $x \in J$. Thus, a suitable choice for $u_{r}(x)$ is

$$
u_{r}(x)=\int_{0}^{x} \frac{W_{q, r}\left(\phi_{1}, \ldots, \phi_{n}\right)(q t)}{W_{q}\left(\phi_{1}, \ldots, \phi_{n}\right)(q t)} \cdot \frac{b(t)}{a_{0}(t)} d_{q} t
$$


and then $\psi_{0}$ has the form

$$
\psi_{0}(x)=\sum_{i=1}^{n} \phi_{i}(x) \int_{0}^{x} \frac{W_{q, i}\left(\phi_{1}, \ldots, \phi_{n}\right)(q t)}{W_{q}\left(\phi_{1}, \ldots, \phi_{n}\right)(q t)} \cdot \frac{b(t)}{a_{0}(t)} d_{q} t
$$

proving formula (4.1).

Example 4.2. Consider the equation

$$
-\frac{1}{q} D_{q^{-1}} D_{q} y(x)+y(x)=b(x)
$$

where $b(\cdot)$ is a continuous function defined in $\mathbb{R}$. The corresponding homogeneous equation is

$$
D_{q}^{2} y(x)-q y(q x)=0 .
$$

A fundamental set of solutions of $(4.4)$ is $\{\sin (x ; q), \cos (x ; q)\}$. Substituting in (4.1) and using $W_{q}(\sin (\cdot ; q), \cos (\cdot ; q))(x) \equiv-1$, every solution of (4.3) has the form

$$
\begin{aligned}
\psi(x)= & c_{1} \sin (x ; q)+c_{2} \cos (x ; q) \\
& -q \int_{0}^{x}(\sin (x ; q) \cos (q t ; q)-\cos (x ; q) \sin (q t ; q)) b(q t) d_{q} t
\end{aligned}
$$

where $x \in \mathbb{R}, c_{1}$ and $c_{2}$ are arbitrary constants.

\section{References}

[1] Abreu, L. D. and Bustoz, J., On the Completeness of Sets of $q$-Bessel Functions. In: Theory and Applications of Special Functions (ded. to M. Rahman; eds.: M. E. H. Ismail et al.). Dev. Math. 13. New York: Springer 2005, pp. 29 - 38.

[2] Abreu, L. D., Bustoz, J. and Caradoso, J. L., The roots of the third Jackson q-Bessel functions. Int. J. Math. Math. Sci. 67 (2003), $4241-4248$.

[3] Abu Risha, M. H., Annaby, M. H., Ismail, M. E. H. and Mansour, Z. S., Existence and uniqueness Theorems of $q$-difference equations (submitted).

[4] Andrews, G. E., Askey, R. and Roy R., Special Functions. Cambridge: Cambridge Univ. Press 1999.

[5] Annaby, M. H. and Mansour, Z. S., Basic Sturm liouville problems. J. Phys. A 38 (2005), 3775 - 3797. Corrigendum: J. Phys. A 39 (2006), 8747.

[6] Annaby, M. H. and Mansour, Z. S., On the zeros of basic finite Hankel transforms. J. Math. Anal. Appl. 323 (2006), $1091-1103$.

[7] Bustoz, J. and Cardoso, J. L., Basic analog of Fourier series on a $q$-linear grid. J. Approx. Theory 112 (2001), 134 - 157. Erratum: J. Approx. Theory, 113 (2001), 326 . 
[8] Exton, H., q-Hypergeometric Functions and Applications. Chichester: EllisHorwood 1983.

[9] Gasper, G. and Rahman, M., Basic Hypergeometric Series. New York: Cambridge Univ. Press 1990.

[10] Ismail, M. E. H., Basic Bessel functions and polynomials. SIAM J. Math. Anal. 12 (1981), $454-468$.

[11] Jackson, F. H., On q-definite integrals. Quart. J. Pure Appl. Math. 41 (1910), $193-203$.

[12] Koelink, H. T. and Swarttouw, R. F., On the zeros of the Hahn-Exton $q$-Bessel function and associated $q$-Lommel polynomials. J. Math. Anal. Appl. 186 (1994), $690-710$.

[13] Shilov, G. E., Linear Algebra. New York: Dover 1971.

[14] Swarttouw, R. F., The Hahn-Exton q-Bessel Funktionen. PhD thesis. Delft: Technical Univ. 1992.

[15] Swarttouw, R. F. and Meijer, H. G., A q-analogue of the Wronskian and a second solution of the Hahn-Exton $q$-Bessel difference equation. Proc. Amer. Math. Soc. 120 (1994)(3), 855 - 864.

Received March 22, 2005; revised June 24, 2005 